\title{
ALCANCE Y LENGUAJE DE LA CIENCIA ${ }^{1}$
}

Soy un objeto físico en un mundo físico. Algunas de las fuerzas de este mundo físico chocan con mi superficie. Los rayos de luz golpean mi retina; moléculas bombardean mis oídos y las puntas de mis dedos. Respondo emanando ondas de aire concéntricas. Estas ondas toman la forma de un torrente de discurso acerca de mesas, personas, moléculas, rayos luminosos, retinas, ondas de aire, números primos, clases infinitas, alegrías, dolores, bien y mal.

Mi capacidad de responder de esta manera compleja consiste en el hecho de que he asimilado una buena parte de la cultura de mi comunidad, y acaso la he modificado y elaborado un poco por mí mismo. A su vez, todo este entrenamiento consiste en una incidencia de fuerzas físicas, fundamentalmente expresiones por parte de otros, sobre mi superficie y en cambios graduales en mi propia constitución que provienen de estas fuerzas físicas. Todo lo que soy o espero poder ser se debe a irritaciones de mi superficie junto con aquellas tendencias latentes para responder que hayan podido encontrarse en mi plasma original. Todo el saber de los tiempos se debe a la irritación de las superficies de una sucesión de personas, de nuevo junto con las condiciones internas iniciales de varios individuos.

Pero, ¿cómo sabemos que nuestro conocimiento debe depender de esta manera y únicamente de la irritación superficial y de las condiciones internas? Solamente porque, de una manera general, sabemos cómo es el mundo, con rayos luminosos, moléculas, hombres, retinas, etc. Es pues nuestro entendimiento del mundo físico, por fragmentario que sea, el que nos permite ver hasta qué punto es limitada la evidencia de la cual se predica este entendimiento. Es nuestro entendimiento, tal cual es, de lo que está más allá de nuestras superficies, lo que muestra nuestra evidencia de que el entendimiento se limita a nuestras superficies. Pero esta reflexión hace surgir ciertos recelos de orden lógico: porque - ¿acaso nuestro hablar mismo de rayos luminosos, moléculas y hombres no es sino sonido y furia in-

1 Este trabajo se presentó en uno de los Congresos bi-centenales de la Universidad de Columbia (octubre de 1954). La versión que se publicó en The Unity of Knowledge (Doubleday, Nueva York, 1955) fue modificada por los editores sólo con el consentimiento previo del autor y sin su conocimiento de los cambios reales. Puesto que en opinión del autor estos cambios hacían perder mucho del sentido original, el texto original se publica aquí con el permiso de los Regentes de la Universidad de Columbia.

[La traducción presente fue hecha a partir del texto publicado por The British Journal for the Philosophy of. Science, vol. VIII, n' 29, 1957.] 
ducidos por la irritación de nuestras superficies, lo cual no significa nada? La visión del mundo que otorgaba plausibilidad a esta modesta relación acerca del mundo es, según esta misma relación, una fabricación sin fundamento.

Pero razonar de este modo es sucumbir a falacia: una falacia especificamente filosófica y de la cual los filósofos se percatan cada vez más. No podemos poner en duda, con sentido, la realidad del mundo exterior o negar que existan evidencias de objetos externos en el testimonio de nuestros sentidos; porque hacerlo equivale a disociar los términos "realidad" y "evidencia" de aquellas aplicaciones mismas que originalmente más contribuyeron a investir a estos términos con cualquier inteligibilidad que para nosotros tengan.

Nos embebemos de filosofía natural arcaica con la leche de nuestras madres. En la madurez, al ponernos al corriente de la literatura actual y al hacer algunas observaciones suplementarias propias, tenemos una idea más clara de las cosas. Pero se trata de un proceso de crecimiento y cambio gradual: no rompemos con el pasado ni alcanzamos criterios de evidencia y realidad distintos en su estilo de los criterios vagos de los niños y los legos. La ciencia no es un sustituto del sentido común, sino una de sus prolongaciones. La búsqueda del conocimiento es, propiamente hablando, un simple esfuerzo por ensanchar y profundizar el conocimiento que el hombre de la calle ya posee, moderadamente, en relación con las cosas que lo rodean. Desconocer el meollo mismo del sentido común, necesitar pruebas para aquello que el físico y el hombre de la calle aceptan como una trivialidad, no sólo no es un perfeccionismo laudable; es una confusión pomposa, un fracaso en observar la fina distinción entre el niño y el agua del baño.

Aceptemos pues la realidad física, ya sea a la manera ingenua del hombre de la calle, ya con un grado mayor o menor de complejidad científica. Al hacerlo nos constituimos en recipientes y portadores del saber evolutivo de los tiempos. Después, prosiguiendo en detalle nuestra teoría de la realidad física así aceptada, sacamos conclusiones con relación, principalmente, a nuestras propias personas físicas, $\mathrm{y}$ aun a nuestras propias personas como portadoras de saber. Una de las conclusiones es que este mismo saber en el cual estamos comprometidos nos ha sido inducido por irritación de nuestras superficies físicas y no de otra manera. He aquí un pequeño caso de saber acerca del saber. Si lo consideramos correctamente no tiende a contravenir el saber acerca de lo cual es saber. Al contrario, nuestra hipótesis inicial y no-crítica de un mundo físico obtiene apoyo pragmático a partir de lo que contribuye a una relación coherente del hecho de portar saber o de otros fenómenos naturales.

Una vez que hemos visto que en nuestro conocimiento del mundo ex- 
terior no podemos basarnos en nada sino en la irritación de superficie, dos preguntas se entremeten —una de ellas buena, la otra mala. La mala, más tarde olvidada, es la pregunta de si, después de todo, existe algún mundo exterior. La buena es ésta: ¿de dónde proviene la fuerza de nuestra noción según la cual existe un mundo exterior?; ¿de dónde nuestra persistencia al representarnos el discurso como algo sobre la realidad, y una realidad más allá de la irritación?

No es que se trate de que el mero acontecer del habla misma se conciba de alguna manera como prueba prima facie de la existencia de una realidad como sujeto. Mucho de lo que decimos es considerado, aun por el hombre de la calle, como irreferencial: "Hola", "Gracias", "Hum, hum", no reclaman ninguna realidad. Son respuestas físicas semánticamente a la par con el reflejo rotular. Pero entonces ¿de dónde proviene la idea de una objetividad científica?; ¿de dónde el que consideremos el lenguaje como ocasionalmente descriptivo de una manera en que no lo son otros estremecimientos de protoplasma irritable?

Esta es una pregunta para la ciencia natural del mundo exterior, en particular para la psicología de los animales humanos. La pregunta contiene dos partes no del todo separables: ¿de dónde proviene la insistencia en un mundo de objetos externos situado frente al lenguaje? y, ¿de dónde la insistencia en un mundo de objetos externos situados frente a uno mismo? En realidad podemos proceder a contestar, de una manera general, esta doble pregunta en forma bastante plausible, sin ningún psicologizar demasiado elaborado.

2

Supongamos que una de las primeras palabras adquiridas por un niño particular es la palabra "rojo". ¿Cómo la aprende? Se le acostumbra a expresiones de la palabra y presentaciones simultáneas de rojo; además, su propio parloteo es aplaudido cuando se aproxima a "rojo" en presencia del rojo. A la larga adquiere el arte de aplicar la palabra de manera ni demasiado estrecha ni demasiado amplia para el gusto de su madre. Este proceso de aprendizaje es familiar bajo muchos nombres: asociación, condicionamiento, entrenamiento, formación de hábitos, refuerzo y extinción, inducción.

Sea lo que fuere lo que descubran nuestros colegas de laboratorio acerca del mecanismo interno de este proceso, podemos estar seguros de esto: su posibilidad misma depende de una tendencia previa en el niño para pesar desigualmente diferencias cualitativas. Lógicamente, mientras $a, b$ y $c$ sean tres y no una, hay exactamente tanta diferencia entre $a$ y $b$ como entre $a$ y $c$; de todos modos, tantas clases dividen a $a$ de $b$ (en otras palabras, 
contienen a una y no a la otra) como a $a$ de $c$. Por otra parte, para el niño, algunas diferencias deben contar más que otras si el proceso descrito para aprender "rojo" tiene que progresar de alguna manera. Ya sea de manera innata, ya como el resultado de aprendizaje pre-lingüístico, el niño debe tener más tendencia a asociar una pelota roja con otra pelota roja que con una pelota amarilla; más tendencia a asociar una pelota roja con una cinta roja que con una azul; y más tendencia a disociar la pelota de sus alrededores que a disociar sus partes entre sí. De otra manera ningún entrenamiento podría modelar el uso que el niño hace de la palabra "rojo", puesto que ninguna ocasión futura sería más fuertemente favorecida por la aplicación anterior que cualquier otra. Una apreciación activa de algo como "tipos naturales" o, por lo menos una tendencia a responder en grados diferentes debe estar presente antes de que se pueda aprender la palabra "rojo".

Así, en el comienzo del aprendizaje del lenguaje, las palabras se aprenden en relación a aquellas semejanzas y contrastes ya apreciados sin el beneficio de la palabra. No es asi sorprendente que atribuyamos estas semejanzas y contrastes a algo real ni que pensemos en el lenguaje como un aparato superimpuesto que permite hablar sobre lo real.

Las semejanzas y contrastes que subrayan nuestro primer aprendizaje del lenguaje no deben tan sólo ser apreciables de manera pre-verbal; deben ser, además, intersubjetivos. La sensitividad hacia la rojez no será de ningún provecho para el niño al aprender "rojo" de su madre, excepto en tanto la madre sea capaz de apreciar que el niño se confronta a algo rojo. De ahí, acaso, nuestras primeras vislumbres de un mundo exterior. Es posible que el más primitivo sentido de la exterioridad sea un sentimiento del refuerzo de semejanzas y contrastes por parte de la madre en las primeras fases del aprendizaje de palabras. Así se siente lo real, primeramente y ante todo, como previo al lenguaje y exterior a uno mismo. Es aquello por lo cual la madre responde y que la madre nombra.

La prioridad de lo no-lingüístico con respecto a lo lingüístico disminuye cuando prosigue el aprendizaje. Se presenta la erudición: es decir, el tipo de aprendizaje que depende de un previo aprendizaje de palabras. Aprendemos "malva" en una edad ya avanzada, por medio de una fórmula del tipo "el color de" o "un color a mitad de camino entre". Y el principio de erudición arraiga pronto; el niño no habrá aprendido todavía muchas palabras antes de que su propio vocabulario venga a figurar como un agente primordial en su acrecentamiento. En este momento el niño es ya capaz de sostener conversaciones rudimentarias en su comunidad estrecha; su conocimiento del lenguaje y del mundo constituyen una masa unitaria.

Sin embargo, quedamos tan poderosamente impresionados por la fase inicial de nuestra educación que continuamos pensando en el lenguaje en general como en un aparato secundario o superimpuesto que nos permite 
hablar acerca de cosas reales. Tendemos a no darnos cuenta de que la mayoría de las cosas y la mayoría de los rasgos de lo que llamamos mundo, se aprenden a través del lenguaje y se cree en ellos mediante una proyección a partir del lenguaje. Así, algunas personas no-críticas alcanzan una teoría del lenguaje como copia: ven los elementos del lenguaje como nombres de elementos de la realidad y al verdadero discurso como un mapa de la realidad. Proyectan sobre el mundo los caprichos del lenguaje de manera indiscriminada, llenando el universo de "ies" y "oes", singulares y plurales, definidos e indefinidos, hechos y estados de cosas, simplemente sobre la base de que hay elementos paralelos y distinciones del lado del lenguaje.

La tarea general que la ciencia se impone es la de especificar cómo es realmente la "realidad": tarea de delinear la estructura de la realidad a diferencia de la estructura de uno u otro de los lenguajes tradicionales (excepto, claro está, cuando la ciencia es la gramática misma). La noción de la realidad independiente del lenguaje, forma parte del hombre de ciencia desde sus primeras impresiones, pero éste evita o minimiza la fácil reificación de los rasgos lingüísticos.

Pero ¿cómo es posible que los hombres de ciencia lleguen a ser de esta manera críticos y discriminadores en cuanto a sus reificaciones? Si todo discurso es una mera respuesta a la irritación de superficie, ¿por qué evidencia puede decirse que la proyección de un mundo por un hombre es más sólida que la de otro? $\mathrm{Si}$, como se ha sugerido antes, los términos "realidad" y "evidencia" deben su inteligibilidad a sus aplicaciones en el sentido común arcaico, ¿por qué no podemos entonces hacer a un lado las presunciones de la ciencia?

La razón por la cual no podemos hacerlo es que la ciencia es ella misma unà continuación del sentido común. El hombre de ciencia es indistinguible del hombre de sentido común en cuanto a su sentido de la evidencia, salvo que el hombre de ciencia es más cuidadoso. Este cuidado acrecentado no es una revisión de los criterios evidenciales, sino solamente la colección y el uso más paciente y sistemático de lo que cualquiera podría considerar como evidente. Si el hombre de ciencia niega a veces lo que un lego supersticioso podría haber llamado evidencia ello puede deberse solamente a que el hombre de ciencia posee otras evidencias contrarias que, si se presentan pacientemente y detalle a detalle al lego, serían consideradas por él como superiores. Puede también suceder que el lego sufra de alguna descuidada cadena de razonamientos, mediante la cual y desde tiempo atrás, llegó a considẹrar ciertos tipos de conexión como evidenciales: cadena errónea en cuanto que un análisis cuidadoso de sus propios pasos mal observados y hace tiempo olvidados sería suficiente para desengañarlo. (Un ejemplo es el de 'la falacia del jugador' - la noción de que cuanto más paga el negro tanto más es probable que salga el rojo.) 
No que el lego posea un criterio explícito de evidencia. Tampoco el científico. El científico empieza con el sentido primitivo de evidencia que como lego posee y lo emplea cuidadosa y sistemáticamente. Con todo, no lo reduce todavía a regla, aunque elabore y emplee variados métodos estadísticos en un esfuerzo por evitar que se le vaya de las manos en casos complejos. Al poner a la naturaleza ante las pruebas más embarazosas que pueda inventar, el hombre de ciencia aprovecha al máximo su olfato lego para la evidencia. Al mismo tiempo agudiza este olfato mismo, haciéndose de una prosbócide artificial de tarjetas perforadas y papel cuadriculado.

Nuestra última pregunta, era, en suma, cómo la ciencia va más allá del sentido común; y la respuesta es, en una palabra: "sistema". El científico introduce sistema en su búsqueda y escrutinio de la evidencia. El sistema, además, dicta las hipótesis mismas del hombre de ciencia: son bienvenidas las que conduzcan más a la simplicidad de la teoría general. A su vez, las predicciones, una vez que han sido deducidas de las hipótesis, están sujetas a la evidencia en turno; pero las hipótesis tienen, como única recomendación, micntras son hipótesis, la consideración de simplicidad sistemática. En este sentido, la simplicidad misma —en algún sentido de este difícil término - cuenta como un tipo de evidencia; y los científicos han tendido desde hace tiempo a ver la más simple de dos hipótesis no sólo como la más agradable sino como la más probable. No supongamos, sin embargo, que por fin hemos encontrado un tipo definitivo de evidencii, aceptable para la ciencia y ajena al sentido común. Al contrario, el hecho de favorecer la hipótesis verosímilmente más simple es un hábito del lego desarrollado por medio de la ciencia. La cuestión de la simplicidad sistemática parece peculiarmente científica en espíritu solamente porque la ciencia es aquello que la ciencia resulta.

La noción de una realidad independiente del lenguaje deriva de las impresiones más primitivas- si las especulaciones de las páginas anteriores son justas- y pasa después a la ciencia con una venganza. Porque el sentido de exterioridad tiene sus raíces, si nuestras especulaciones son correctas, en la intersubjetividad tan esencial para el aprendizaje del lenguaje; y la intersubjetividad es vital no sólo para el lenguaje sino también para las empresas, también sociales, de la ciencia. Todos los hombres deben ser testigos ante los datos de la ciencia, y las verdades de la ciencia deben ser verdaderas sin que importe quien las pronuncie. La ciencia se ha desarrollado más con masas y velocidades que con agrados y desagrados. $\mathrm{Y}$ así, cuando la ciencia confronta agrados y desagrados lo hace bajo la forma del comportamiento, intersubjetividad observable. El lenguaje en general es robustamente extravertido, pero la ciencia lo es todavía más. 
Sería racionalismo infundado suponer que podemos señalar el asunto de la ciencia antes de hacer ciencia y de alcanzar cierto cuerpo de teoría. Considérese así, por pura analogía, la tarea más pequeña de definir el asunto de la química. Habiendo seguido la química podemos describirla ex post facto como el estudio de las combinaciones de átomos y moléculas. Pero una delimitación tan precisa del asunto de la química no sería posible hasta que este asunto estuviera ya realizado en buena medida. Ahora bien, la situación es semejante cuando se trata de la ciencia en general. Describir la ciencia como el dominio de los juicios cognoscitivos no nos dice nada porque el definiens necesita aquí urgentemente clarificarse como el definiendum. Aprovechando el trabajo científico existente y sin tener escrúpulos de identificarnos con una postura científica sustantiva, podemos, sin embargo, delinear hasta cierto punto el objeto científico, o el dominio cognoscitivo. Esta incapacidad de ser capaces de formular una tarea hasta no haberla realizado a medias es cosa trivial.

El pensamiento, en cuanto es de menor complejidad, es inseparable del lenguaje - ciertamente en la práctica y muy probablemente en principio. La ciencia, aun cuando busque rasgos de realidad independientes del lenguaje, no puede hacer a un lado el lenguaje ni aspirar a la neutralidad lingǘstica. Hasta cierto punto, con todo, el hombre de ciencia puede realzar la objetividad y disminuir la interferencia del lenguaje por la elección de este mismo lenguaje. $Y$ nosotros, preocupados por poner al desnudo la esencia del discurso científico, podemos volver a trabajar el lenguaje de la ciencia más allá de lo que pudiera exigírsele razonablemente al científico practicante. Veremos ahora esta operación.

Así, en el espíritu del esquematismo filosófico y no en el de la reforma lingüística, podemos empezar por eliminar las que se conocen como palabras indicadores (Goodman) o particulares egocéntricos (Russell) : "yo", "tú", "esto", "aquello", "aqui", "ahora", "entonces", y otros por el estilo. Es claro que tenemos que hacer esto si las verdades de la ciencia deben ser literalmente verdaderas con independencia del autor o el lugar de la expresión. Sólo así, en verdad, llegamos a poder hablar de sentencias, es decir, ciertas formas lingǘsticas, como verdaderas o falsas. Mientras se mantienen los indicadores no es la sentencia sino solamente los diversos acontecimientos de su expresión lo que puede ser llamado verdadero o falso. ${ }^{2}$

Además de los indicadores, una fuente frecuente de fluctuación, por lo que a la verdad se refiere, es normalmente la ambigüedad. Una y la misma frase, en cuanto forma lingüística, puede ser verdadera en un caso y falsa en otro porque la ambigüedad de una palabra se resuelve en ella de manera distinta por las circunstancias concomitantes en ambas ocasiones.

2 Conservamos la palabra sentencia y no frase porque sentencia expresa más claramente el sentido lógico del texto. [Trad.] 
La frase ambigua "Your mother bore you" puede explicitarse de una manera cuando sigue las huellas de una frase como " $x$ bore you" y de otra cuando sigue las huellas de una frase de la forma " $x$ bores you". ${ }^{3}$

En las lenguas indoeuropeas existe todavía una tercera y conspicua fuente de fluctuación en cuanto a la verdad y la falsedad, a saber, el tiempo. En realidad es sólo una variante del fenómeno de las palabras-indicadores: los tiempos pueden parafrasearse en términos de verbos sin tiempo gobernados por el indicador "ahora", o por "antes de ahora", etc.

¿Cómo evitar las palabras indicadores? Podemos recurrir a nombres personales o a descripciones en lugar de "yo" y "tú", a fechas o descripciones equivalentes en lugar de "ahora", y a nombres de lugar o descripciones en vez de "aquí". Podría objetarse que, en última instancia, es inevitable el uso de algo equivalente al uso de los indicadores. Pero ésta no es una objeción seria; lo que importa es la subsecuente posibilidad de evitar indicadores; lo que importa es que es posible en principio expresar la ciencia en una notación tal que ninguna de sus sentencias fluctúe entre la verdad y la falsedad de una expresión a otra. Los términos primitivos o irreductibles desde el punto de vista de esta notación científica pueden todavía sernos solamente inteligibles a través de explicaciones en un lenguaje ordinario con indicadores, tiempo y ambigüedad. En cualquier caso el lenguaje científico es una esquirla del lenguaje ordinario, no un sustituto.

Aceptando así que podemos eliminar indicadores de la ciencia, ¿qué fin se persigue con ello? En primer lugar, cierta objetividad compatible con el temperamento científico: la verdad se vuelve invariante en relación al hablante y a la ocasión. Al mismo tiempo se sirve un fin más urgente: el de simplificar y facilitar un departamento básico de la ciencia, a saber, la lógica deductiva. Porque, considérense los cánones muy elementales de deducción que conducen a " $p$ y $q$ " a " $p$ " y de " $p$ " a " $p$ o $q$ " y de " $p$ y si $p$ entonces $q$ " a " $q$ ". La letra " $p$ ", que significa cualquier sentencia, aparece dos veces en cada una de estas reglas; y es claro que las reglas dejan de ser sólidas si la frase que ponemos por "p" puede ser verdadera en uno de los casos y falsa en el otro. Pero, formular leyes lógicas de tal manera que no dependan así de la suposición de una verdad y una falsedad fijas sería decididamente extraño y complicado así como también carente de cualquier resultado.

En la práctica ciertamente no eliminamos de nuestro trabajo científico indicadores, tiempos y ambigüedad, ni limitamos nuestro empleo de la lógica a sentencias así purificadas. En la práctica meramente suponemos todos que estos puntos de variación son fijos dentro del espacio de nuestra argumentación lógica; no tenemos que recurrir a la paráfrasis explícita,

3 Conservamos en inglés el ejemplo. La palabra "bore" puede significar "aburrir" 0 , cuando es pretérito de "to bear", "dar a luz". [Trad.] 
excepto en los puntos donde variaciones locales de contexto dentro del argumento lógico mismo amenazan con equívocos.

Este procedimiento práctico se racionaliza muchas veces aceptando de hecho entidades abstractas, "proposiciones", dotadas de la precisión y fijeza requeridas de que carecen las frases mismas, para después decir que las leyes de la lógica tienen que ver con las proposiciones y no con sus burdas incorporaciones sentenciales. Pero esta aceptación solamente conduce a oscuridad. Hay menos misterio en imaginar una forma idealizada de lenguaje científico en el cual las sentencias se construyan de tal modo que nunca vacilen entre la verdad y la falsedad. Es significativo que el discurso científico tienda en realidad hacia este ideal en la misma proporción en que la ciencia está desarrollada. Disminuyen las ambigüedades y las deformaciones de lugar y época. El tiempo, en particular, da lugar a un tratamiento cuadridimensional del espacio-tiempo.

Una forma básica para las sentencias de la ciencia puede entonces representarse como ' $F a$ ' donde ' $a$ ' toma el lugar de un término singular que se refiere a algún objeto entre aquellos que existen según la teoria científica en cuestión y ' $F$ ' representa un término general o predicado. La frase ' $F a$ ' es verdadera si y sólo si el objeto cumple con el predicado. No debe leerse ningún tiempo en la predicación ' $F a^{\prime}$; cualquier fecha pertinente debe ser integral más que referirse a los términos representados por ' $F$ ' y por ' $a$ '.

Las frases compuestas se construyen a partir de tales predicaciones con la ayuda de conectivas lógicas y operadores familiares: ' $y$ ', 'no', el cualificador universal ' $(x)^{\prime}$ ' ("cada objeto $x$ es tal que") y el cuantificador existencial ' $(\exists x)$ ' ("por lo menos un objeto $x$ es tal que"). Un ejemplo sería ' $(x)$ ' no ( $F x$ y no $G x)$ ' que dice que ningún objeto $x$ es tal que $F x$ y no $G x$; en suma, todo $F$ es un $G$.

Se dirá que un término singular dado y un término general o predicado dado corresponden si el término general es verdad de solamente un objeto, a saber, el objeto al cual el término singular se refiere. Un término general que de esta manera corresponda a un término singular será, naturalmente, "de extensión singular", es decir, verdadero exactamente de un objeto; pero, con todo, pertenecerá a la categoría gramatical de los términos generales representada más por la ' $F$ ' que por la ' $a$ ' de ' $F a$ '. Ahora bien, la totalidad de la categoría de los términos singulares puede ser eliminada en favor de los términos generales con vistas a la economía: a saber, los términos generales que corresponden a estos términos singulares. Porque si ' $a$ ' representa a cualquier término singular y ' $F$ ' a cualquier término general correspondiente '..$x \ldots$ ' representará lo que hayamos querido afirmar conteniendo ' $a$ '. Entonces podemos dispensarnos de ' $a$ ' y afirmar ' $(\exists x) \quad(F x$ y 
$\ldots x \ldots)$ '. Es claro que esto será verdad si y sólo si '...a...' era verdadero. Si queremos proseguir diciendo explícitamente que el objeto que cumple con ' $F$ ' es único podemos hacerlo fácilmente así:

$$
\text { (x) (y) no }[F x \text { y } F y \text { y no }(x=y)]
$$

siempre que el signo de identidad ' $=$ ' esté en nuestro vocabulario.

Podrá preguntarse: ¿cómo podemos estar seguros de que habrá un término general que corresponda a un término singular dado? La cuestión puede ser vista así: meramente re-analizamos lo que habían sido términos singulares como términos generales de extensión singular y lo que había sido una referencia-a como una verdad-de, y lo que había sido '...a...' como ' $(\exists x) \quad(F x$ y $\ldots x \ldots)$ '. Si el viejo término singular era un nombre propio aprendido ostensivamente, se lo re-analiza como un término general aprendido de manera similar.

La referencia reciente a ' $=$ ' nos recuerda que los términos generales relativos, o predicados poliádicos, deben ser permitidos junto a los monádicos: es decir, las frases de nuestro lenguaje científico regimentado comprenderán no sólo ' $F x^{\prime}$, ' $F y$ ', ' $G x^{\prime}$, etc., sino también ' $H x y$ ', ' $H z x$ ', 'Jyz', $K x y z$ ' y otros parecidos para los predicados propiamente interpretados ' $F$ ', ' $G$ ', ' $H$ ', ' $J$ ', ' $K$ ', etc. (de donde ' $H$ ' en particular podría interpretarse como ' $=$ '). El resto de las sentencias se construye a partir de estas atómicas por medio de ' $y$ ', 'no', (' $x$ '), ' $(y)$ ', etc. Los términos singulares, lo hemos visto, pueden dejarse de lado. También pueden dejarse de lado los cuantificadores existenciales ' $(\exists x)$ ', ' $(\exists y)$ ', etc., puesto que ' $\mathrm{B}(x)$ ' puede parafrasearse así: 'no (x) no'.

Además de los términos singulares simples hay que contar con operadores, como ' + ', que llevan consigo términos singulares complejos como ' $x+y$ '. Pero no es difícil ver como éstos pueden eliminarse en favor de predicados poliádicos correspondientes - por ejemplo, un predicado ' $\Sigma$ ' tal que ' $\Sigma z x y$ signifique que $z$ es $x+y$ '.

Este patrón para un lenguaje científico es, evidentemente, algo confinador. No existen en él palabras de objetos. Además, no existen sentencias dentro de sentencias, salvo en contextos de conjunción, negación y cuantificación. Pero con todo es suficiente de manera muy general como un medio de teoría científica. Mucho o todo de lo que es probable que se desee en una ciencia puede ajustarse a esta forma a fuerza de construcciones de variable ingeniosidad, familiares para los estudiosos de la lógica. Para tomar tan sólo el más trivial y familiar de los ejemplos considérese el modismo 'si-entonces': puede manejarse sustituyendo "si $p$ entonces $q$ ' por 'no ( $p$ y no $q)$ '.

Puede ser instructivo profundizar un momento en este ejemplo. Es noto- 
rio que "no $(p$ y no $q)$ ' no es una traducción de "si $p$ entonces $q$ ' ni debe pretender serlo. El punto es que en los lugares donde por lo menos en matemáticas y en otros trabajos típicamente científicos, encontramos que donde ordinariamente emplearíamos 'si-entonces' podemos arreglárnoslas perfectamente bien con la forma sustituta 'no ( $p$ y no $q$ ), a veces aumentado de un cuantificador universal. No preguntamos si nuestro modismo reformado constituye de algún modo un análisis semántico genuino del viejo modismo; sólo nos encontramos con que dejamos de depender del viejo modismo en nuestro trabajo técnico. Aquí vemos, paradigmáticamente, el contraste entre el análisis lingüístico y la construcción de teorías.

\section{5}

Las variables ' $x$ ', ' $y$ ', etc., adjuntas a la notación de cuantificación, entrañan un ensanchamiento de la noción de sentencia. Una sentencia que contenga una variable sin cuantificador (por ejemplo, ' $F x^{\prime} \circ(y) F x y^{\prime}$, carente de ' $(x)$ ') no es una sentencia en el sentido ordinario de verdadero-falso. Es verdadero para algunos valores de sus variables libres, tal vez, y falso para otras. Llamada sentencia abierta es afín más bien a un predicado: en lugar de tener un valor de verdad (verdad o falsedad) puede decirse que posee una extensión, siempre que se conciba a ésta como la clase de las valoraciones en variables libres para las cuales es verdad. Para mayor comodidad se habla también de la extensión de una sentencia cerrada, pero lo que entonces se entiende es simplemente su valor de verdad.

Una sentencia compuesta que contiene una sentencia como cláusula componente es llamada contexto extensional de esta sentencia componente si, al suplantar el componente por cualquier sentencia con la misma extensión, el compuesto permanece incambiado en cuanto a su propia extensión. En el caso especial en el cual las sentencias referidas son sentencias cerradas, los contextos son extensionales si todas las sustituciones de verdades por componentes verdaderos y de falsedades por componentes falsos dejan a los contextos verdaderos, verdaderos, y a los falsos, falsos. En suma, en el caso de sentencias cerradas con los contextos extensionales son lo que comúnmente se conoce como funciones de verdad.

Es bien sabido y fácilmente visible que los medios conspicuamente limitados que nos hemos permitido para componer sentencias - por ejemplo, ' $y$ ', 'no' y cuantificadores - son solamente capaces de generar contextos extensionales. Resulta, por otra parte, que no nos limitan más que esto: las únicas maneras de encajar sentencias dentro de sentencias que se entremezclan y resisten el análisis en términos de ' $y$ ', 'no' y cuantificadores muestran ser contextos de otro tipo que el tipo extensional. Será instructivo examinarlos. 
Es claro que una cita es, según nuestros criterios, no-extensional. No podemos libremente colocar verdades por verdades y falsedades por falsedades en una cita sin afectar el valor de verdad de una sentencia más amplia de la cual la cita forme parte. La cita puede, sin embargo, siempre hacerse a un lado en favor del deletreo. En lugar, por ejemplo de:

Heráclito dijo 'panta rei'

'panta rei' contiene tres sílabas,

podemos decir (con Tarski) :

Heráclito dijo: pi-alfa-nu-tau-alfa espacio ro-épsilon-iota,

Y correspondientemente para el otro ejemplo, proporcionándonos así nombres de letras junto a un guión a modo de signos de concatenación. Ahora bien, mientras la versión citatoria mostraba una sentencia (la sentencia griega), encajada en otra, la versión basada en el deletreo no lo muestra; así pues no existe ya aquí la cuestión de la extensionalidad.

En cualquiera de las dos versiones, estamos hablando de cierto objeto -una forma lingǘstica- con la ayuda, como siempre, de un término singular que se refiere a este objeto. La cita produce un término singular con un fin; el deletreo, con otro fin. La cita es una especie de escritura pictórica, conveniente en la práctica; pero el deletreo es el que mejor proporciona el análisis apropiado para una teoría lógica de los signos.

Vimos que los términos singulares nunca se necesitan totalmente. Los términos singulares que se encuentran en el deletreo en particular pueden naturalmente eliminarse por fin en favor de una notación del tipo de las consideradas en páginas recientes, en las cuales solamente hay predicados, cuantificadores, variables, 'y' y 'no'. El guión de concatenación cede entonces su lugar a un predicado triádico análogo a ' $\Sigma$ ' del párrafo 4 , y los términos singulares 'pi', 'alfa', etc., ceden ante términos generales que "corresponden" a ellos en el sentido del párrafo 4.

Un contexto más seriamente no-extensional es el discurso indirecto: "Heráclito dijo que todo fluye". Esto no es, como era el caso de la cita, una sentencia acerca de una forma lingüística específica y nombrable. Tal vez, contrariamente a la línea seguida en el caso de la citación, debamos aceptar el discurso indirecto como algo que entraña el caso no-extensional de una sentencia en otra. De ser así, el discurso indirecto se resintiria al esquematismo antes adelantado para el lenguaje científico.

Es así tanto más interesante pensar que el discurso indirecto está en cualquier caso de acuerdo con la característica objetividad de la ciencia. Se trata de un modismo subjetivo. Mientras que las citas relatan un acontecimiento externo del habla o de la escritura mediante una descripción de la 
forma escrita o el sonido hablado observables, el discurso indirecto relata el acontecimiento en términos más bien de una proyección subjetiva de uno mismo en el estado de ánimo imaginado de quien habla o de quien escribe. El discurso indirecto es una cita menos la objetividad y la precisión. El ordenamiento de la evidencia del discurso indirecto remite a la cita.

Es significativo que la latitud de paráfrasis permisible en el discurso indirecto nunca haya sido fijada; y es todavía más significativo que tan pocas veces se sienta la necesidad de hacerlo. Fijarlo sería un movimiento científico, movimiento científicamente inmotivado en cuanto el discurso indirecto tiende a alejarse de la objetividad que la ciencia busca.

El discurso indirecto, en la forma común de "dice que", es la cabeza de una familia que incluye también "cree que", "duda de que", "se sorprende de que", "desea que", "lucha por", y otras semejantes. De la subjetividad apuntada en el caso de "dice que" participan doblemente estos otros modismos porque lo que éstos describen, en términos de una proyección subjetiva de uno mismo, no es ni tan sólo la conducta del protagonista del habla, sino, a su vez, su estado subjetivo.

Otros casos de modismos no-extensionales, fuera de la familia inmediatamente arriba enumerada, son "porque" y el fenómeno estrechamente relacionado del condicional contrario-a-los-hechos. Ahora bien, es un hecho irónico pero familiar el que a pesar de que el asunto de la ciencia sea descriptible en lenguaje no-científico como el descubrimiento de causas, la noción misma de causa no tenga un lugar firme en la ciencia. La desaparición de la terminología causal de la jerga de una y otra rama de la ciencia ha parecido señalar el progreso en el entendimiento de las ramas respectivas. Por consiguiente además del citar, que ya vimos como debe tratarse, los varios modismos familiares no-extensionales tienden a alejarse de lo que mejor tipifica al espíritu científico. No que debieran o pudieran generalmente ser evitados en el discurso cotidiano ni aún en la ciencia entendida ampliamente, sino que sus usos disminuyen de valor en la medida en que los enunciados de la ciencia se vuelven más explícitos y objetivos. Empezemos a ver como la forma lingüística esquematizada en el párrafo 4 podría, a pesar de sus estrechas. limitaciones, ser suficiente para la ciencia en su estado más puro.

En tanto nos adherimos a este esquematismo idealizado, pensamos en la ciencia como en algo que comprende aquellas verdades expresables en términos de "y", "no", cuantificadores, variables y ciertos predicados apropiados a la ciencia en cuestión. En esta enumeración de materiales podrá parecer que tenemos una aproximación a un posible criterio de lo que cuenta como "puramente cognoscitivo". Pero el criterio, a pesar de todo su aparente 
carácter estricto, es todavía demasiado flexible. Para especificar una ciencia dentro del molde descrito tenemos que saber además cuáles deben ser los predicados, y cuál ha de ser el dominio de objetos donde se sitúan las variables de cuantificación. No todos los modos de resolver estos detalles congeniarán con los ideales científicos.

Si miramos a la ciencia actual como un asunto en marcha, podemos fijar de manera general el dominio de los objetos. Los objetos físicos -habitantes del espacio-tiempo- pertenecen claramente a ella. Esta categoría comprende sin discriminación lo que antiguamente se habría distinguido como sustancias y como modos o estados de las de otras sustancias. Un hombre es objeto de cuatro dimensiones que, puede extenderse a una dimensión temporal de 83 años. Cada parte espacio-temporal del hombre cuenta como otro objeto cuatri-dimensional más pequeño. Tal sería el caso de un presidente electo durante dos meses. Un ataque de fiebre es otro caso, si por mor a la claridad ontológica lo identificamos, como podemos hacerlo convenientemente, con su víctima durante la duración del ataque.

Al contrario de la creencia popular tal ontología física da también cabida a los estados mentales. Una inspiración o una alucinación pueden, como el ataque de fiebre, identificarse con su huésped mientras duran. La viabilidad de esta identificación de cualquier ataque mental $x$, que corresponde a la rebanada de tiempo $x^{\prime}$ de su huésped físico, puede verse al reflexionar acerca de la siguiente sencilla maniobra. Cuando $P^{\prime}$, aplicada a $x$, es cualquier predicado que queremos aplicar a $x$, expliquemos $P^{\nu}$ como verdadero de $x^{\prime}$ si y sólo si $P$ es verdad de $x$. Este paralelismo, junto a la extensionalidad del lenguaje científico, nos permite eliminar la antigua $P$ y la antigua $x$ de nuestra teoría y proseguir solamente con $P^{\prime}$ y $x^{\prime}$ rebautizadas como $P$ y $x$. Tal es, en efecto, la identificación. Deja a nuestros modismos mentalistas bastante intactos pero los reconcilia con una ontología física.

Este fácil fisicalismo de los estados mentales no descansa de modo alguno en una teoría del paralelismo entre los impulsos nerviosos -0 las concentraciones químicas - y la recurrencia de especies predeterminadas de estados mentales. Podría muy bien ser que desde ahora y para siempre la única manera de adivinar si un hombre está inspirado, deprimido, engañado o adolorido, consistiera en preguntárselo o en observar su comportamiento general; no examinando sus reacciones nerviosas aunque fuese con instrumentos de una sutileza jamás soñada. El descubrimiento del paralelismo sugerido sería un gran logro científico, pero el fisicalismo de que aqui se habla no lo requiere.

Esta fisicalización no es, en verdad, suficiente para que la ciencia acepte “inspiración", "alucinación", “dolor" ni otros términos mentalistas. Aun cuando éstos se vuelvan términos generales concretos aplicables a objetos físicos (por ejemplo, tajadas temporales de personas) pueden, algunos de ellos, se- 
guir demasiado vagos para la utilidad científica. Términos de disposiciones y otros predicados que no se prestan a una verificación inmediata no son impermisibles por sí mismos; pero entre ellos los hay mejores o peores. Cuando la tajada temporal de una persona debe clasificarse bajo el rótulo de la inspiración o de la alucinación y cuando no debe hacerse puede ser una cuestión demasiado indecisa para cualquier propósito útil. Pero lo que entonces importa es la aceptabilidad de ciertos predicados y no la de ciertos objetos, valores, de variables de cuantificación.

No abandonemos este último tema todavía: el de la ontología o el valor que pueda otorgarse a las variables. Como hemos visto, podemos ir lejos con los objetos físicos. Pero no sabemos que basten. Ciertamente, como acabamos de mostrarlo, no necesitamos incluir objetos mentales. Pero necesitamos incluir objetos abstractos, si debemos acomodarnos a la ciencia tal como está constituida. Algunas de las cosas que queremos decir en la ciencia pueden obligarnos a admitir en la gama de valores de las variables de cuantificación no solamente objetos físicos sino también clases y relaciones entre ellos; también números, teoría de los números, y otros objetos de la matemática pura. Porque las matemáticas - no las matemáticas ininterpretadas sino la genuina teoría de los conjuntos, la lógica, la teoría de los números, el álgebra de los números reales y complejos, el cálculo diferencial e integral, etc.- se sitúan mejor cuando se ven como parte integral de la ciencia, como en la física, la economía, etc., en las cuales se dice que las matemáticas reciben su aplicación.

Las indagaciones acerca del fundamento de las matemáticas han puesto en claro que todas las matemáticas en el sentido aquí empleado pueden reducirse a la lógica y a la teoría de los conjuntos y que los objetos necesarios para las matemáticas en este sentido pueden reducirse a una sola categoría, la de las clases -incluyendo clases de clases, clases de clases de clases, etc. Nuestra provisional ontología para la ciencia, nuestra serie provisional de valores para las variables de la cuantificación, se reducen así a lo que sigue: objetos físicos, clases de ellos, clases, a su vez, de los elementos de este dominio combinado y así de manera ascendente.

Hemos alcanzado el presente estadio en nuestra caracterización del marco de la ciencia no por razonamientos a priori a partir de la naturaleza de la ciencia en cuanto ciencia, sino más bien recogiendo aspectos de la ciencia de nuestros días. Los rasgos especiales así explorados incluyen la noción de objeto físico, el concepto cuadridimensional del espacio-tiempo, el molde clásico de las matemáticas clásicas modernas, la orientación verdad-falsedad de la lógica común $\mathrm{y}$, en verdad, la extensionalidad misma. Uno $\mathrm{u}$ otro de estos rasgos podría muy bien cambiar al avanzar la ciencia. La noción de un objeto físico como porción intrínsecamente determinada del continuo espacio-temporal encuadra ya dudosamente con los desarrollos modernos de la mecánica 
cuántica. Existen sabios que incluso sugieren que los descubrimientos de la mecánica cuántica podrían entenderse mejor revisando la misma dicotomía verdad-falsedad.

En cuanto, por fin, a la cuestión de los predicados admisibles, en general podemos estar seguros de que un predicado se prestará a la empresa científica solamente si está relativamente libre de vaguedad en algunos respectos cruciales. Si el predicado es de tal tipo que deba básicamente usarse en la aplicación a los objetos macroscópicos del sentido común, será claramente útil que exista una tendencia general al acuerdo, entre los observadores, por lo que toca a su aplicación a estos objetos; porque en tales aplicaciones la verificabilidad intersubjetiva reside en la verificabilidad intersubjetiva de los datos de la ciencia. En el caso de un predicado principalmente aplicable a los objetos físicos remotos de la observación o del sentido común, lo que se requiere, por otra parte, es que estén meramente libres de las vaguedades que oscurecen su función científica. Pero decir esto es meramente decir que los predicados apropiados para la ciencia son los que hacen expedito el propósito de confirmación intersubjetiva y la claridad y simplicidad teórica. Estos mismos propósitos gobiernan también la decisión ontológica - la determinación del alcance de la cuantificación-; porque, claramente, la presente ontología tentativa de los objetos físicos y de las clases se abandonará inmediatamente cuando encontremos una alternativa que sirva mejor para estos propósitos.

En la ciencia todo es provisional, todo admite revisión -incluso, como hemos visto, la ley del tercero excluido. Pero la ontología, hasta nuevas revisiones, está más controlada que lo que puede llamarse la ideologia —la cuestión de los predicados admisibles. Hemos encontrado una ontología provisional en los objetos físicos, y en las clases, pero el léxico de los predicados queda decididamente abierto. Que la ontología sea relativamente definida, hasta nueva revisión, es cosa requerida por la mera presencia de los cuantificadores en el lenguaje de la ciencia; porque puede decirse que los cuantificadores han sido interpretados y entendidos sólo en cuanto hemos establecido el alcance de las variables. $Y$ es algo implícito en un teorema de matemáticas que el fondo de predicados debería estar para siempre sujeto a suplementación; porque es sabido que para cualquier teoría, por rica que sea, existen clases que no son la extensión (párrafo 5) de ninguna de sus sentencias.

\author{
W. V. QUINE \\ (Trad. de Ramón Xirau)
}

